\title{
Determination of granulometrical composition of the clinker by grinding in a ball mill to determine the specific consumption of additional energy
}

\author{
Cristian Ciobanu ${ }^{1}$, Paula Tudor $^{3}$, Gabriel-Alexandru Constantin ${ }^{2, *}$ and Gabriel Musuroi ${ }^{2}$ \\ ${ }^{1}$ CEPROCIM Bucharest, Romania \\ ${ }^{2}$ University Politehnica of Bucharest, Faculty of Biotechnical Systems Engineering, Romania \\ ${ }^{3}$ University Politehnica of Bucharest, Faculty of Entrepreneurship, Business Engineering and \\ Management, Romania
}

\begin{abstract}
This paper presents results regarding the grinding behaviour of two batches of material (clinker) in a ball mill with its own two-step grinding process: the first one with a ball load, the second with a load of cylindrical metal bodies. The first step was performed until the residue $\mathrm{R}_{009}$ has reached aprox. $35 \%$, and the second stage until Blaine's specific surface was over $3800 \mathrm{~cm}^{2} / \mathrm{g}$. Periodically, at 10 -minute intervals, the fineness of the clinker and the specific energy consumption were determined. These consumptions are quantified from the first determination and relates to the weight of the material subjected to grinding ( $20 \mathrm{~kg}$ clinker / from a source), determining the grinding ability of the material.
\end{abstract}

\section{Introduction}

The cement consists of dispersed solid particles whose particles have a size between 0.1 and $250 \mu \mathrm{m}$. Particle size, particle size distribution, but also the composition of the cement has a special influence on its behaviour during wetting, on the development of microstructures and on the properties of concrete or cement-based materials. It is important that Portland cement particle sizes fall within certain limits so that they are properly moistened and to form a material with a good subsequent behaviour. More, Excessive grinding leads to additional energy consumption (sometimes unjustified), and insufficient grinding can affect the binder properties of the cement particles and their distribution in the prepared material.

Among the properties that have been examined through experiments and computer simulation are: setting time, heat release, capillary porosity percolation, diffusivity, chemical shrinkage, autogenous shrinkage, internal relative humidity evolution, and interfacial transition zone microstructure, [1]. The authors concluded that, for lower watercement ratio values, coarser cements can be used that can provide equivalent or even superior performance, which would reduce the production costs to the manufacturer.

* Corresponding author: constantin.gabriel.alex@gmail.com 
Still, there are researchers who claim that cement particles larger than $45 \mu \mathrm{m}$ are difficult to hydrate, and also the largest of $75 \mu \mathrm{m}$ cannot be completely hydrated, when analysing the rate of reactivity and the development of stresses in the prepared concrete, [2]. Author of this paper, [2], states, however, that estimates of reactivity rates for similar cement compositions cannot be made without knowing the particle size distribution by sedimentation methods. In any case, there must be a correlation between the setting time and the fineness of the cement.

The standard method for determining particle size and particle size distribution is ASTM C115-96, with a limit of detection of a smaller size of $7.5 \mu \mathrm{m}$ [3]. This is based on the turbidimetric method for determining the fineness of the material. The Electrical Zone Sensing (EZS) method, which is based on the Coulter principle, can operate in the particle size range between $0.2(0.6)-800 \mu \mathrm{m}$, while laser diffraction is used successfully when most particles have dimensions below $1 \mu \mathrm{m}$, [3].

From the four techniques analysed by the authors of the previous paper: laser diffraction (LAS), detection of electrical areas (EZS), X-ray gravitational sedimentation (XRS) and scanning electron microscopy (SEM), laser diffraction (with both wet and dry dispersion), gave the best yields, this being the basis for determining the specific Blaine surface.

For cements, are two systems for determining fineness, namely, specific surface Blaine (air permeability) and turbidimetry. Blaine fineness is an indirect measure of the total surface of each cement sample and can be determined according to ASTM C204-11 "Standard Test Method for Fineness of Hydraulic Cement by Air Permeability Apparatus".

For Portland cement from the same source, the heat of hydration is affected by the fineness of the cement and the particle size distribution. Grinding to a greater fineness Blaine, in correlation with decreasing particle size, leads to an increase in the heat of hydration, experimentally confirmed [4].

The necessary fineness of the final cement is due to the milling means of the ball mills and the size distribution of the feed material. To increase the efficiency of ball mills, for optimum distribution of the final material according to the particle size, it is necessary, therefore, a correlation between the size of the balls and the size of the particles when feeding the mill, [5]. It was concluded that a ternary or at least binary mixture of balls of different sizes $(50+20+10 \mathrm{~mm})$ leads to a final material of optimum size.

More energy efficient grinding technologies, applied in the grinding of cement, are described comparatively in the paper [6], from the point of view of operating principles, grinding efficiency, specific energy consumption, the production capacity and the quality of the cement obtained. Other papers addressing the theme of milling process with balls dedicated to grinding cement, including its control are [7-11]

The present paper presents the results regarding the grinding behaviour of two batches of material (clinker) in a ball mill with its own two-step grinding process: the first one with a ball load, the second with a load of cylindrical metal bodies.

\section{Material and method}

Energy consumption between the moments when the fineness of the material is determined (in our case the consumption was read directly on the electricity meter) was determined using a wattmeter. These consumptions have accumulated since the beginning of the determination and have been related to the mass of the batch $(20 \mathrm{~kg}$ clinker/factory from 2 factories), calculating the specific energy consumption $\mathrm{w}_{1 \mathrm{i}}$. The aptitude index for milling is the specific energy consumption $\mathrm{w}_{1}$ corresponding to a reference fineness and $\mathrm{w}$ - the specific energy consumption of the industrial mill, [12]. 


$$
c_{1}=\frac{w}{w 1}
$$

In fig. 1 shows the scheme of a laboratory mill and the working process of a ball mill.
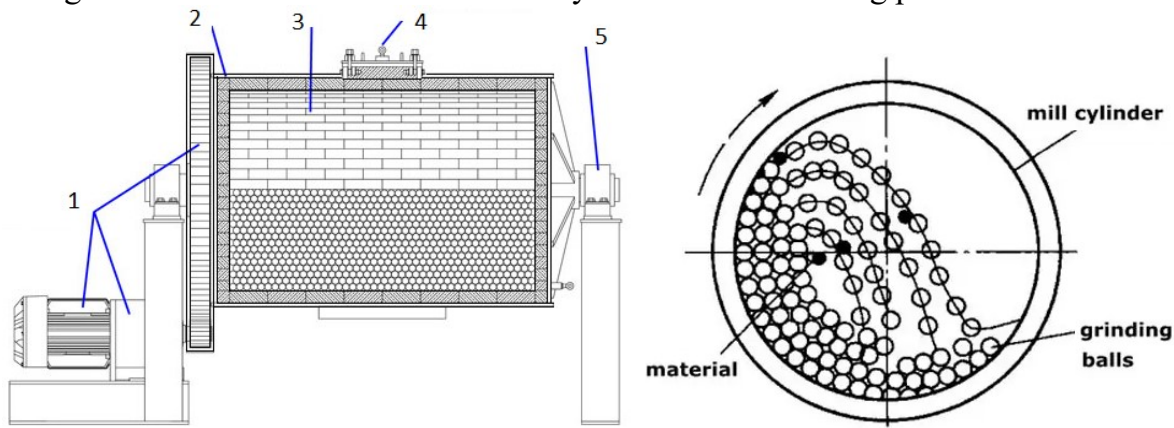

Fig.1 Schematic ball mill, [13]

a): ball mill diagram; 1- motor, gear reducers and ring and pinion gear; 2- cylinder; 3- brick lining; 4- discharge hatch; 5- bearings; b): working process of ball mill

To determine the specific surface (SSB) of the grinded material we chose the method of the Blaine permeability meter. The Blaine procedure is applicable for all cements defined in the standard EN 196-6 :2018, [14].

$a$.

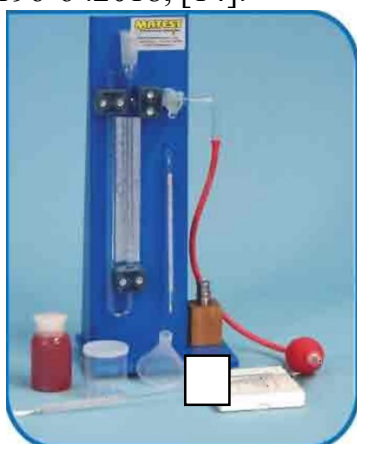

c.

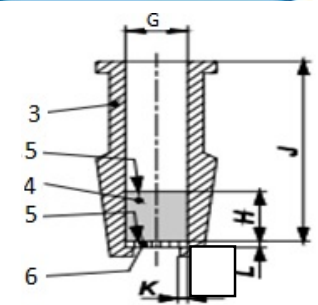

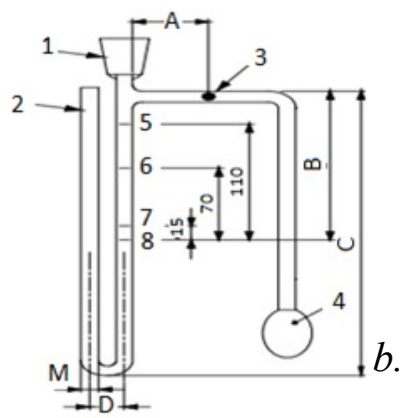
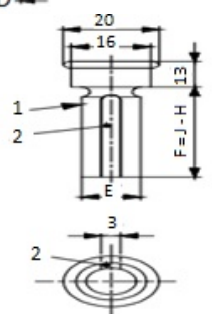

$d$.

Fig.2 Permeabilimeter Blaine, [14,15]: a)General aspect; b)Manometer: 1- conical socket; 2manometer; 3-stopcock; 4-rubber bulb; 5-8-etched lines; c)Permeability cell: 3-cell; 4-cement bed compacted by the plunger; 5- paper filters; 6- perforated disc; d)Plunger piston: 1-piston body; 2flat

The Blaine permeability meter also includes: stopwatch; analytical balance; manometric liquid; apparatus for determining the density of cement (Le Chatelier $100 \mathrm{ml}$ quoted balloon).

The specific surface S was calculated accordingly SR EN 196-6:2018 [16] and is conventionally expressed in $\mathrm{cm}^{2} \cdot \mathrm{g}^{-1}$, as it is:

$$
S=\frac{K}{\rho} \cdot \frac{\sqrt{e^{3}}}{(1-e)} \cdot \frac{\sqrt{t}}{\sqrt{10 \eta}}
$$


where: $K$ - the constant of the device, e - porosity of the layer; $\mathrm{t}$ - time measured (s); $\rho$ bulk density of the material, $\left(\mathrm{g} \cdot \mathrm{cm}^{-3}\right), \eta$ - the viscosity of the air at the test temperature, according to table no.1, $(\mathrm{Pa} \cdot \mathrm{s})$

With the specified porosity e $=0.500$ and the test temperature $20 \pm 2{ }^{\circ} \mathrm{C}$ :

$$
S=\frac{52.43 \cdot K \sqrt{t}}{\rho}
$$

where: $K$ thus determined:

$$
K=1.414 \cdot S_{o} \cdot \rho_{o} \frac{\sqrt{10 \eta_{o}}}{\sqrt{t_{o}}}
$$

$\mathrm{S}_{0}$ - the specific surface of the reference cement, $\left(\mathrm{cm}^{2} \cdot \mathrm{g}^{-1}\right), \rho_{0}$ - the bulk density of the reference cement, $\left(\mathrm{g} \cdot \mathrm{cm}^{-3}\right), \mathrm{t}_{0}$ - the average of three timed values of time, $(\mathrm{s}), \mathrm{\eta}_{0}$ - the viscosity of the air corresponding to the average of three temperatures, $(\mathrm{Pa} \cdot \mathrm{s})$ and is given according to table 1 .

Table 1. Bulk density of $\mathrm{Hg}$, viscosity of the air $\eta_{0}$ and $\sqrt{10 \eta_{0}}$ function of temperature, [16]

\begin{tabular}{|c|c|c|c|}
\hline $\begin{array}{c}\text { Temperature, } \\
{ }^{\mathbf{0}} \mathbf{C}\end{array}$ & Bulk density of $\mathbf{H g}, \boldsymbol{\rho}_{\mathbf{H g}}\left[\mathbf{g} \cdot \mathbf{c m}^{-\mathbf{3}}\right]$ & Viscosity of the air $\boldsymbol{\eta}_{\mathbf{0}}, \mathbf{P a} \cdot \mathbf{s}$ & $\sqrt{\mathbf{1 0} \times \boldsymbol{\eta}_{\mathbf{0}}}$ \\
\hline 16 & 13.560 & 0.00001800 & 0.013416 \\
\hline 17 & 13.560 & 0.00001805 & 0.013435 \\
\hline 18 & 13.550 & 0.00001810 & 0.013454 \\
\hline 19 & 13.550 & 0.00001815 & 0.013472 \\
\hline 20 & 13.550 & 0.00001819 & 0.013487 \\
\hline 21 & 13.540 & 0.00001824 & 0.013506 \\
\hline 22 & 13.540 & 0.00001829 & 0.013524 \\
\hline 23 & 13.540 & 0.00001834 & 0.013543 \\
\hline 24 & 13.540 & 0.00001839 & 0.013561 \\
\hline \multicolumn{3}{|c|}{ Intermediate values must be obtained by linear interpolation } \\
\hline
\end{tabular}

For the determination of the laser particle size analysis for Clincher A and F samples, the Mastersizer 2000E (MALVERN, UK) was used, [16].

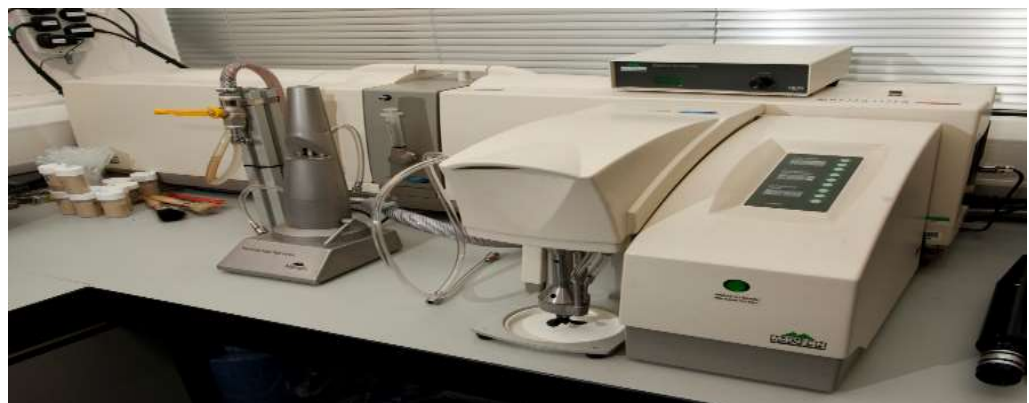

Fig.3 Mastersizer 2000E (MALVERN, UK) for laser particle size distribution, [16]

Laser particle size distribution was performed under the following measurement conditions: Wet dispersion (dispersion liquid: Ethanol $\approx 850 \mathrm{ml}$ ); Refractive index dispersion liquid: 1.360; Ultrasound duration: $60 \mathrm{sec}$; Ultrasound level: $10 \mu \mathrm{m}$; Stirring pump speed: $2300 \mathrm{rpm}$; Laser diffraction apparatus: Mastersizer 2000E; Focal distance of the lenses:2.35; dimensional range: $0.100 \mu \mathrm{m}-1000.00 \mu \mathrm{m}$; Optical concentration: $18.45 \%$; Light dispersion model applied : MieAbsorbtie theory: 0.1 . 


\section{Results and discussions}

Following the determinations were recorded the following energy consumption and specific surface Blaine (SSB) for two types of clinker A and F from the 2 cement factories (Table 2).

Table 2. Specific consumption and Blaire surface area for the two types of clinker

\begin{tabular}{|c|c|c|c|c|c|c|c|c|c|c|c|}
\hline 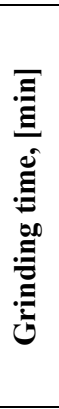 & 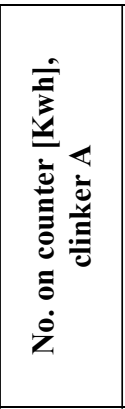 & 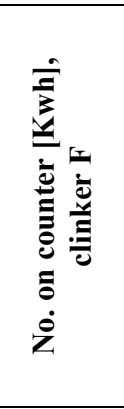 & 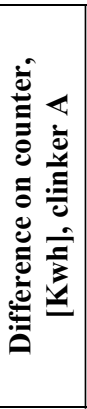 & 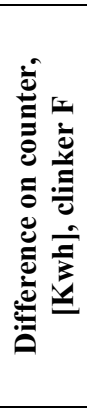 & 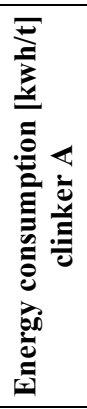 & 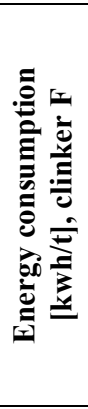 & 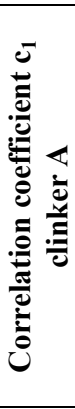 & 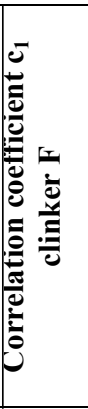 & 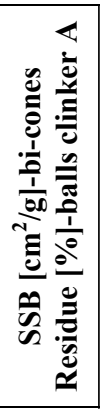 & 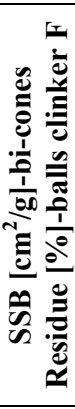 & 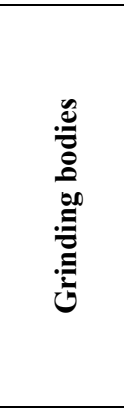 \\
\hline 0 & 7350421 & 7356265 & 0 & 0 & - & - & - & - & - & - & $\begin{array}{c}\text { Grinding } \\
\text { balls }\end{array}$ \\
\hline 10 & 7350661 & 7356504 & 240 & 239 & - & - & - & - & $\begin{array}{c}51.68 \\
\% \\
\end{array}$ & $\begin{array}{c}53.4 \\
\% \\
\end{array}$ & $\begin{array}{c}\text { Grinding } \\
\text { balls }\end{array}$ \\
\hline 20 & 7350882 & 7356729 & 221 & 225 & - & - & - & - & $33.6 \%$ & $\begin{array}{c}34.5 \\
\%\end{array}$ & $\begin{array}{c}\text { Grinding } \\
\text { balls }\end{array}$ \\
\hline 30 & 7351096 & 7356946 & 214 & 217 & 10.7 & 10.85 & 3.08 & 3.03 & 2250 & 2190 & Bi-cones \\
\hline 40 & 7351314 & 7357167 & 218 & 221 & 10.9 & 11.05 & 3.02 & 2.98 & 2650 & 2640 & Bi-cones \\
\hline 50 & 7351526 & 7357387 & 212 & 220 & 10.6 & 11 & 3.11 & 2.99 & 2830 & 2960 & Bi-cones \\
\hline 60 & 7351746 & 7357617 & 220 & 230 & 11 & 11.5 & 2.99 & 2.86 & 3180 & 3130 & Bi-cones \\
\hline 70 & 7351968 & 7357837 & 222 & 220 & 11.1 & 11 & 2.97 & 2.99 & 3520 & 3290 & Bi-cones \\
\hline 80 & 7352196 & 7358069 & 228 & 232 & 11.4 & 11.6 & 2.89 & 2.84 & 3590 & 3580 & Bi-cones \\
\hline 90 & 7352417 & 7358290 & 221 & 221 & 11.05 & 11.05 & 2.98 & 2.98 & 3700 & 3870 & Bi-cones \\
\hline 100 & 7352642 & - & 225 & - & 11.25 & - & 2.93 & - & 3870 & - & Bi-cones \\
\hline
\end{tabular}

Total energy consumption clinker $A=\frac{7352642-7350421}{20}=111.05 \mathrm{kwh} / \mathrm{t}$

Total energy consumption clinker $F=\frac{7358290-7356265}{20}=101.25 \mathrm{kwh} / \mathrm{t}$

$\mathrm{W}_{\text {industrial mill consumption }}=32.92 \mathrm{kWh} / \mathrm{t}$ according to the value calculated by the cement factory.

Regarding energy consumption, Atmaca and Kanoglu found that this consumption is $24.75 \mathrm{kWh} /$ ton farine, depending on the temperature and humidity of the material, [17].

Results regarding laser chemical and particle size distribution of the material subjected to grinding (clinker A and F) are presented in Table 3.

Table 3. Chemical analysis results clinker A and $\mathrm{F}$

\begin{tabular}{|c|c|c|c|}
\hline \multirow{2}{*}{ Determination } & \multirow{2}{*}{ U.M. } & Test code & Test code \\
\cline { 3 - 4 } & & Clinker A & Clinker F \\
\hline Loss on calcination - L.C. & $\%$ & 0.53 & 0.96 \\
\hline $\mathrm{SiO}_{2}$ & $\%$ & 21.20 & 20.88 \\
\hline $\mathrm{Al}_{2} \mathrm{O}_{3}$ & $\%$ & 5.66 & 5.04 \\
\hline $\mathrm{Fe}_{2} \mathrm{O}_{3}$ & $\%$ & 2.99 & 3.80 \\
\hline $\mathrm{CaO}$ & $\%$ & 66.34 & 66.78 \\
\hline
\end{tabular}




\begin{tabular}{|c|c|c|c|}
\hline $\mathrm{MgO}$ & $\%$ & 1.20 & 0.80 \\
\hline $\mathrm{SO}_{3}$ & $\%$ & 0.83 & 0.74 \\
\hline $\mathrm{Na}_{2} \mathrm{O}$ & $\%$ & 0.21 & 0.12 \\
\hline $\mathrm{K}_{2} \mathrm{O}$ & $\%$ & 0.50 & 0.34 \\
\hline $\mathrm{CaO}_{\text {free }}$ & $\%$ & 0.31 & 0.92 \\
\hline $\mathrm{HCl}-\mathrm{Na}_{2} \mathrm{CO}_{3}$ & $\%$ & 0.15 & 0.17 \\
\hline
\end{tabular}

Following the measurements the following charts were obtained.

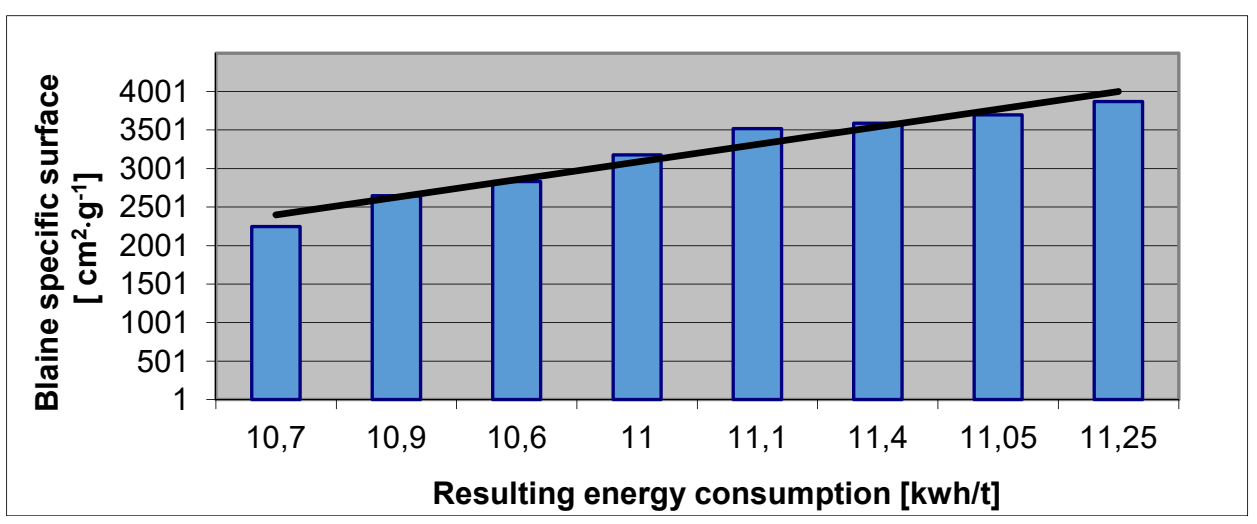

Fig.4 Grinding aptitude index clinker A

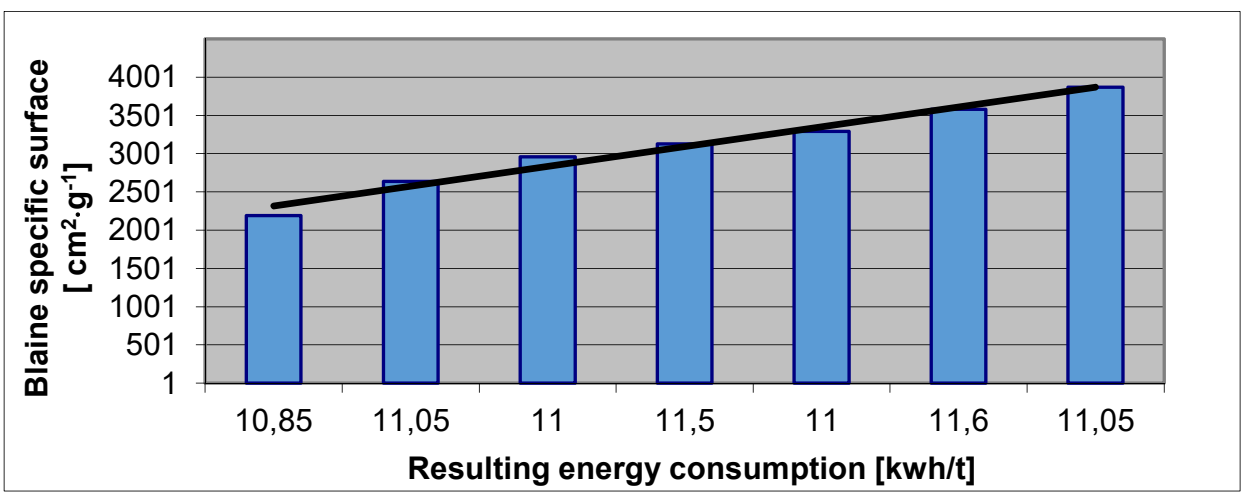

Fig.5 Grinding aptitude index clinker F

Based on the equilibrium diagrams, the basic composition of the clinkers was calculated. The calculation was made in the hypothesis of solidification at thermodynamic equilibrium and at thermodynamic nonequilibrium. In case of solidification at full equilibrium, the glass phase is completely absent. In reality, no thermodynamic balance is achieved at solidification and the mineralogical composition calculated from such a hypothesis can be considered as a potential composition, that is, possible, understanding by this that the respective chemical composition is not equal to the composition of the real minerals. Assuming the clinker as being placed in the thermal equilibrium subsystem $\mathrm{C}_{3} \mathrm{~S}$ $\mathrm{C}_{2} \mathrm{~S}-\mathrm{C}_{3} \mathrm{~A}-\mathrm{C}_{4} \mathrm{AF}\left(\mathrm{MA}_{\mathrm{i}}>0.64\right)$, Bogue has established the following calculation formulas for mineralogical constituents, [12]:

$$
\begin{aligned}
& \% \mathrm{C}_{4} \mathrm{AF}=3.04 \% \mathrm{Fe}_{2} \mathrm{O}_{3} \\
& \% \mathrm{C}_{3} \mathrm{~A}=2.65 \% \mathrm{Al}_{2} \mathrm{O}_{3}-1.69 \% \mathrm{Fe}_{2} \mathrm{O}_{3}
\end{aligned}
$$




$$
\begin{aligned}
& \% \mathrm{C}_{3} \mathrm{~S}=4.07 \% \mathrm{CaO}-7.60 \% \mathrm{SiO}_{2}-6.72 \% \mathrm{Al}_{2} \mathrm{O}_{3}-1.42 \% \mathrm{Fe}_{2} \mathrm{O}_{3} \\
& \% \mathrm{C}_{2} \mathrm{~S}=8.60 \% \mathrm{SiO}_{2}+5.06 \% \mathrm{Al}_{2} \mathrm{O}_{3}+1.07 \% \mathrm{Fe}_{2} \mathrm{O}_{3}-3.05 \% \mathrm{CaO}
\end{aligned}
$$

Thus, from the mineralogical composition according to Bogue's calculation, the values presented in table 4 resulted.

Table 4. Mineralogical composition according to Bogue's calculation for two clinkers

\begin{tabular}{|c|c|}
\hline Clinker A & Clinker $\mathbf{~}$ \\
\hline $\mathrm{C}_{3} \mathrm{~S}=66.48$ & $\mathrm{C}_{3} \mathrm{~S}=71.39$ \\
\hline $\mathrm{C}_{2} \mathrm{~S}=11.48$ & $\mathrm{C}_{2} \mathrm{~S}=6.8$ \\
\hline $\mathrm{C}_{3} \mathrm{~A}=9.95$ & $\mathrm{C}_{3} \mathrm{~A}=6.93$ \\
\hline $\mathrm{C}_{4} \mathrm{AF}=9.09$ & $\mathrm{C}_{4} \mathrm{AF}=11.55$ \\
\hline
\end{tabular}

It is observed that $\mathrm{C}_{2} \mathrm{~S}$ clinker $\mathrm{A}>\mathrm{C}_{2} \mathrm{~S}$ clinker $\mathrm{F}(11.48>6.8)$ which results in clinker A requiring additional energy consumption in the mill because it has a much higher content of $\mathrm{C}_{2} \mathrm{~S}$.

The results of the determinations of the laser particle size distribution for clinker A are presented in Table 5.

\begin{tabular}{|c|c|c|c|c|c|c|c|c|c|}
\hline $\begin{array}{c}\text { Granul } \\
\text { ometric } \\
\text { class } \\
(\mu \mathrm{m})\end{array}$ & $\begin{array}{c}\text { Cumula } \\
\text { tive } \\
\text { passing } \\
\text { (\% vol) }\end{array}$ & $\begin{array}{c}\text { Granulo } \\
\text { metric } \\
\text { class } \\
(\mu m)\end{array}$ & $\begin{array}{c}\text { Cumulativ } \\
\text { e passing } \\
\text { (\% vol) }\end{array}$ & \begin{tabular}{|c|} 
Granul \\
ometric \\
class \\
$(\mu \mathrm{m})$ \\
\end{tabular} & $\begin{array}{c}\text { Cumula } \\
\text { tive } \\
\text { passing } \\
(\% \text { vol })\end{array}$ & \begin{tabular}{|c|} 
Granul \\
ometric \\
class \\
$(\mu \mathrm{m})$ \\
\end{tabular} & \begin{tabular}{|c|} 
Cumula \\
tive \\
passing \\
(\% vol)
\end{tabular} & $\begin{array}{c}\text { Granul } \\
\text { ometric } \\
\text { class } \\
(\mu \mathrm{m}) \\
\end{array}$ & $\begin{array}{c}\text { Cumula } \\
\text { tive } \\
\text { passing } \\
\text { (\% vol) }\end{array}$ \\
\hline 0.10 & 0.00 & 3.00 & 12.26 & 13.00 & 42.36 & 64.00 & \begin{tabular}{|c|}
93.99 \\
\end{tabular} & 175.00 & 100.00 \\
\hline 0.20 & 0.00 & 3.15 & 12.99 & 14.00 & 44.32 & 70.00 & 95.79 & 200.00 & 100.00 \\
\hline 0.30 & 0.00 & 3.50 & 14.66 & 15.00 & 46.21 & 75.00 & 96.96 & 225.00 & 100.00 \\
\hline 0.40 & 0.00 & 4.00 & 16.95 & 16.00 & 48.05 & 80.00 & 97.87 & 250.00 & 100.00 \\
\hline 0.50 & 0.35 & 4.50 & 19.13 & 18.00 & 51.58 & 85.00 & 98.59 & 280.00 & 100.00 \\
\hline 0.60 & 0.95 & 5.00 & 21.16 & 20.00 & 54.92 & 90.00 & 99.14 & 315.00 & 100.00 \\
\hline 0.70 & 1.60 & 5.50 & 23.06 & 22.00 & 58.10 & 95.00 & 99.52 & 355.00 & 100.00 \\
\hline 0.80 & 2.21 & 6.00 & 24.83 & 24.00 & 61.12 & 96.00 & 99.58 & 400.00 & 100.00 \\
\hline 0.90 & 2.77 & 6.30 & 25.84 & 25.00 & 62.58 & 100.00 & 99.78 & 450.00 & 100.00 \\
\hline 1.00 & 3.28 & 6.50 & 26.49 & 28.00 & 66.71 & 106.00 & 99.94 & 500.00 & 100.00 \\
\hline 1.20 & 4.16 & 7.00 & 28.06 & 31.50 & 71.10 & 110.00 & 99.99 & 560.00 & 100.00 \\
\hline 1.40 & 4.98 & 7.50 & 29.53 & 32.00 & 71.69 & 115.00 & 100.00 & 600.00 & 100.00 \\
\hline 1.50 & 5.38 & 8.00 & 30.93 & 36.00 & 76.10 & 120.00 & 100.00 & 650.00 & 100.00 \\
\hline 1.60 & 5.79 & 8.50 & 32.26 & 40.00 & 79.95 & 125.00 & 100.00 & 700.00 & 100.00 \\
\hline 1.80 & 6.63 & 9.00 & 33.53 & 45.00 & 84.06 & 128.00 & 100.00 & 750.00 & 100.00 \\
\hline 2.00 & 7.51 & 9.50 & 34.76 & 48.00 & 86.17 & 130.00 & 100.00 & 800.00 & 100.00 \\
\hline 2.20 & 8.42 & 10.00 & 35.94 & 50.00 & 87.45 & 140.00 & 100.00 & 850.00 & 100.00 \\
\hline 2.50 & 9.84 & 11.00 & 38.19 & 55.00 & 90.24 & 150.00 & 100.00 & 900.00 & 100.00 \\
\hline 2.60 & 10.33 & 12.00 & 40.32 & 60.00 & 92.51 & 160.00 & 100.00 & 950.00 & 100.00 \\
\hline 2.80 & 11.29 & 12.50 & 41.35 & 63.00 & 93.65 & 170.00 & 100.00 & 1000.0 & 100.00 \\
\hline
\end{tabular}

Table 5. Particle size distribution for clinker A

The sample subjected to the laser particle size analysis was the material passed through the sieve of $1000 \mu \mathrm{m}$. Residue value on sieve of $1000 \mu \mathrm{m}$ it was of $\mathrm{R}_{1000}=0.0 \%$. Following the results obtained, the chart of the cumulative passage of material was drawn (\% vol) depending on the granulometric class $(\mu \mathrm{m})$ from $0.10 \mu \mathrm{m}$ to $1000 \mu \mathrm{m}$ (see fig. 6 ). 


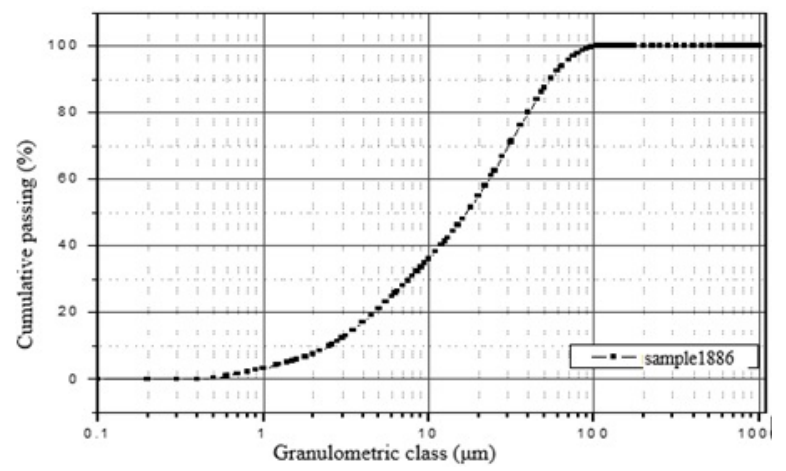

Fig.6 Chart clinker A

The results of the determinations of the laser particle size distribution for clinker $\mathrm{F}$ are presented in Table 6.

Table 6. Particle size distribution for clinker F

\begin{tabular}{|c|c|c|c|c|c|c|c|c|c|}
\hline $\begin{array}{c}\text { Granu } \\
\text { lometri } \\
\text { c class } \\
(\mu m)\end{array}$ & $\begin{array}{l}\text { Cumul } \\
\text { ative } \\
\text { passing } \\
\text { (\% vol) }\end{array}$ & $\begin{array}{c}\text { Granu } \\
\text { lometri } \\
\text { c class } \\
(\mu m)\end{array}$ & $\begin{array}{l}\text { Cumul } \\
\text { ative } \\
\text { passing } \\
\text { (\% vol) }\end{array}$ & $\begin{array}{c}\text { Granu } \\
\text { lometri } \\
\text { c class } \\
(\mu m)\end{array}$ & $\begin{array}{c}\text { Cumula } \\
\text { tive } \\
\text { passing } \\
\text { (\% vol) }\end{array}$ & \begin{tabular}{|c|} 
Granu \\
lometri \\
c class \\
$(\mu m)$
\end{tabular} & $\begin{array}{c}\text { Cumul } \\
\text { ative } \\
\text { passing } \\
\text { (\% vol) }\end{array}$ & $\begin{array}{c}\text { Granu } \\
\text { lometri } \\
\text { c class } \\
(\mu \mathrm{m})\end{array}$ & $\begin{array}{c}\text { Cumul } \\
\text { ative } \\
\text { passing } \\
\text { (\% vol) }\end{array}$ \\
\hline 0.10 & \begin{tabular}{|l|}
0.00 \\
\end{tabular} & 3.00 & \begin{tabular}{|l|}
10.79 \\
\end{tabular} & 13.00 & 41.44 & 64.00 & \begin{tabular}{|l|}
93.24 \\
\end{tabular} & 175.00 & 100.00 \\
\hline 0.20 & 0.00 & 3.15 & 11.48 & 14.00 & 43.47 & 70.00 & 95.04 & 200.00 & 100.00 \\
\hline 0.30 & 0.00 & 3.50 & 13.08 & 15.00 & 45.43 & 75.00 & 96.24 & 225.00 & 100.00 \\
\hline 0.40 & 0.00 & 4.00 & 15.32 & 16.00 & 47.33 & 80.00 & 97.21 & 250.00 & 100.00 \\
\hline 0.50 & 0.30 & 4.50 & 17.49 & 18.00 & 50.97 & 85.00 & 97.99 & 280.00 & 100.00 \\
\hline 0.60 & 0.87 & 5.00 & 19.54 & 20.00 & 54.41 & 90.00 & 98.61 & 315.00 & 100.00 \\
\hline 0.70 & 1.49 & 5.50 & 21.47 & 22.00 & 57.66 & 95.00 & 99.10 & 355.00 & 100.00 \\
\hline 0.80 & 2.08 & 6.00 & 23.29 & 24.00 & 60.75 & 96.00 & 99.19 & 400.00 & 100.00 \\
\hline 0.90 & 2.59 & 6.30 & 24.32 & 25.00 & 62.22 & 100.00 & 99.50 & 450.00 & 100.00 \\
\hline 1.00 & 3.04 & 6.50 & 24.99 & 28.00 & 66.40 & 106.00 & 99.84 & 500.00 & 100.00 \\
\hline 1.20 & 3.79 & 7.00 & 26.60 & 31.50 & 70.80 & 110.00 & 99.97 & 560.00 & 100.00 \\
\hline 1.40 & 4.45 & 7.50 & 28.13 & 32.00 & 71.39 & 115.00 & 100.00 & 600.00 & 100.00 \\
\hline 1.50 & 4.77 & 8.00 & 29.58 & 36.00 & 75.76 & 120.00 & 100.00 & 650.00 & 100.00 \\
\hline 1.60 & 5.10 & 8.50 & 30.96 & 40.00 & 79.55 & 125.00 & 100.00 & 700.00 & 100.00 \\
\hline 1.80 & 5.79 & 9.00 & 32.28 & 45.00 & 83.55 & 128.00 & 100.00 & 750.00 & 100.00 \\
\hline 2.00 & 6.52 & 9.50 & 33.55 & 48.00 & 85.61 & 130.00 & 100.00 & 800.00 & 100.00 \\
\hline 2.20 & 7.31 & 10.00 & 34.77 & 50.00 & 86.85 & 140.00 & 100.00 & 850.00 & 100.00 \\
\hline 2.50 & 8.56 & 11.00 & 37.11 & 55.00 & 89.56 & 150.00 & 100.00 & 900.00 & 100.00 \\
\hline 2.60 & 9.00 & 12.00 & 39.32 & 60.00 & 91.77 & 160.00 & 100.00 & 950.00 & 100.00 \\
\hline 2.80 & 9.89 & 12.50 & 40.39 & 63.00 & 92.89 & 170.00 & 100.00 & 1000.0 & 100.00 \\
\hline
\end{tabular}

The sample subjected to the laser particle size analysis was the material passed through the sieve of $1000 \mu \mathrm{m}$. Residue value on sieve of $1000 \mu \mathrm{m}$ it was of $R_{1000}=0.0 \%$. Following the results obtained, the chart of the cumulative passage of material was drawn (\% vol) depending on the granulometric class $(\mu \mathrm{m})$ from $0.10 \mu \mathrm{m}$ to $1000 \mu \mathrm{m}$ (see fig.7). 
Fig.7 Chart Clinker F

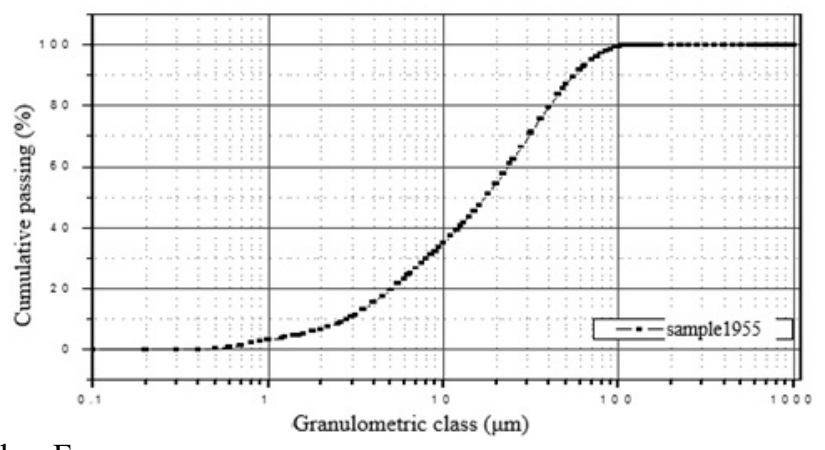

\section{Conclusions}

Cement production requires large amounts of thermal and electrical energy. Energetic efficiency, defined as the total thermal energy consumed per ton of clinker produced and, respectively as the total electricity consumed per ton of cement produced, depends almost entirely on the technology applied in the production process.

According to the laboratory analyses it is observed that the mineralogical composition according to Bogue's calculation $\mathrm{C}_{2} \mathrm{~S}$ clinker $\mathrm{A}>\mathrm{C}_{2} \mathrm{~S}$ clinker $\mathrm{F}(11.48>6.8)$ which results in clinker $\mathrm{A}$ requiring additional energy consumption in the mill because it has a much higher content of $\mathrm{C}_{2} \mathrm{~S}$ (calcium silicate) fact shown by the energy consumption to clinker $\mathrm{A}$ $111.05 \mathrm{kwh} / \mathrm{t}>$ clinker F $101.25 \mathrm{kwh} / \mathrm{t}$.

Results for laser particle size distribution for clinker A and for clinker $\mathrm{F}$ they showed that both clinkers at granulometric class of $115 \mu \mathrm{m}$, has a cumulative passing (\%material volume) is integral $(100 \%)$. Besides reducing production costs, increasing energy efficiency contributes positively to the conservation of natural resources (fuels) as well as reducing greenhouse gas emissions $\left(\mathrm{CO}_{2}\right)$ generated both by combustion of fuels in the clinker oven (direct emissions) or in power plants for the production of electricity consumed in the cement factory (indirect emissions)

A direction of increasing energy efficiency is represented by the use of alternative fuels from waste, method with significant potential for reducing conventional fuel consumption.

Reduction of electricity consumption per tonne of product contributes to the refurbishments made in recent years at cement plants in Romania, which include:

- Replacing air compressors with some efficient ones

- Replacement of old pneumatic cement transport installations

- Resizing the ball mill load and installing systems to optimize their operation

- Installation of a high energy efficiency separator and a state-of-the-art filter

- Optimization of control of the machine flows through the Process Control System.

The paper was funded both from the project "Sustainable development of the base of agromechanical applications in gardens, solariums, vineyards and orchards (DEZDURPRA)", CNFIS-FDI-2020-0184, from the Ministry of Education and Research through the Executive Agency for Financing Higher Education, Research, Development and Innovation, as well as from the European Social Fund within the Sectoral Operational Program Human Capital 2014-2020, through the Financial Agreement of the project "Scholarships for entrepreneurial education between doctoral students and postdoctoral researchers (Be Entrepreneur!)", Contract no. 51680 / 09.07.2019 - SMIS Code: 124539 . 


\section{References}

1. D.P. Bentz, E.J. Garboczi, C.J, Haecker, O.M. Jensen, Cement and Concrete Research, (October 1999), 16 pages, doi: 10.1016/S0008-8846(99)00163-5;

2. D. Kim, Advances in Materials Science and Engineering, (2018), ID 1763524, 6 pages, https://doi.org/10.1155/2018/1763524;

3. C. Ferraris, V. Hackley, A. I. Avilés, Cement Concrete and Aggregates, (December 2004), 11 pages, doi: 10.1520/CCA11920;

4. A. Zayed, Effects of portland cement particle size on heat of hydration, Contract No. BDK84 977-13, Final Report, (December 2013), Florida Department of Transportation, Research Center;

5. N. Hlabangana, G. Danha, E. Muzenda, South African Journal of Chemical Engineering, vol.25, (2018), pag.79-84;

6. O. Genc, Energy - Efficient technologies in cement grinding, Chapter in book „High Performance Concrete Technology and Applications", (2016), doi: 10.5772/64427;

7. S. Mohanty, K. Gupta, S. Raju, Procedia Computer Science, vol.58, (2015), pp.217224;

8. V. Bulgakov, S. Pascuzzi, S. Ivanovs, G. Kaletnik, V. Yanovich, Biosystems engineering, vol. 171, (2018), pag.155- 164;

9. D.C. Tsamatsoulis, Brazilian Journal of Chemical Engineering, (2014), vol. 31, no. 01, pp. 155-170;

10. A. Jankovic, W. Valery, E. Davis, Cement grinding optimisation, Minerals Engineering, vol. 17, (2004), pp.1075-1081

11. J.R.G. Sarduy, J.P.M. Yanes, M.E.G. Rodríguez, J.L.Q. Ferreira and Y.M. Torres, Determining cement ball mill dosage by artificial intelligence tools aimed at reducing energy consumption and environmental impact, Ingeniería e Investigación, Vol. 33, No. 3, (2013), pp.49-54

12. S. Opris, Manual of engineers from cement industry, vol. I, Ed. Tehnica (1994);

13. *** Pictures from CEPROCIM SA, B-dul Preciziei Nr.6, sector 6, Bucuresti;

14. *** SR EN 196-6:2018, Methods for cement testing. Part 6: Fineness determination;

15. *** SR EN 196-2:2013, Methods for cement testing. Part 2:Chemical analysis of cements;

16. $* * *$ https://www.n8equipment.org.uk/equipment/detail/mastersizer-2000e;

17. A. Atmaca, M. Kanoglu, Reducing energy consumption of a raw mill in cement industry, Energy, Vol. 42, Iss. 1, 2012, pp. 261-269 